Check for updates

Cite this: Mater. Adv., 2021, 2,3378

Received 17th December 2020, Accepted 1st April 2021

DOI: 10.1039/d0ma00991a

rsc.li/materials-advances

\section{Polymer micro-particles formed by thiol-ene suspension polymerization using canola oil as a diluent solvent $\dagger$}

\author{
Jared S. Cobb, Chipo Chapusha, Jui Gaikwad, Joshua Michael (D) and \\ Amol V. Janorkar (D) *
}

\begin{abstract}
This work demonstrates the use of canola oil as a biologically compatible solvent for a thiol-ene driven suspension polymerization that effectively creates micron-sized particles. A 1:1 thiol to alkene functional group stoichiometry was maintained for all formulations. Thymol was incorporated into these particles during synthesis as a naturally derived, lipophilic drug with known anti-microbial properties and use in agriculture as a pesticide. UV-Vis spectroscopy demonstrated the removal of surfactant and any excess reagents to be $99 \%$ effective. Particle size was dependent on stirring speed with faster stirring resulting in smaller particles. The release of thymol from the selected ten formulations was measured with UV-Vis spectroscopy at a wavelength of $275 \mathrm{~nm}$ for ten days and was shown to be tailorable by altering the amount of crosslinker and surfactant used during the creation of the particles. A smaller molar ratio of 2-functional thiol monomer compared to 4-functional thiol monomer (0.1:0.9) crosslinker and a higher molar ratio of 2 -functional alkene monomer compared to mono-functional alkene surfactant $(0.9: 0.1)$ resulted in the slower release of thymol. A higher molar ratio of 2-thiol to 4-thiol monomer $(0.8: 0.2)$ and a lower molar ratio of 2 -alkene to surfactant $(0.5: 0.5)$ resulted in faster-releasing particles. The Peppas-Sahlin equation used to model the thymol release found that a super case II drug release mechanism that relies on physical interactions between the polymer chains determined the amount of thymol released. The utility of canola oil as a solvent for a polymer particle delivery system holds promise for their use in environmentally sensitive applications such as a pesticide carrier and anti-microbial products.
\end{abstract}

\section{Introduction}

Essential oils have found many uses in the agricultural, cosmetic, and anti-microbial industries as natural methods to control microorganisms and several invasive pest species. ${ }^{1,2}$ Many essential oils are regarded as safe for human applications, and some have shown broad-spectrum antibiotic, anti-fungal, and anti-viral properties through several mechanisms. ${ }^{3-5}$ Most notably, thymol, a monoterpene, forms a highly reactive phenolic radical that can attack and disrupt the membrane of bacteria. ${ }^{6}$ The biggest problems facing the use of essential oils are their high volatility with no control of their release rate, and their poor water solubility limiting the dosage that can be delivered in biological applications. ${ }^{7}$ Polymer particles are particularly well suited to

Biomedical Materials Science, University of Mississippi Medical Center $2500 \mathrm{~N}$ State Street, Jackson, MS 39216, USA.E-mail: ajanorkar@umc.edu; Fax: 601-984-6087; Tel: 601-984-6170

$\dagger$ Electronic supplementary information (ESI) available. See DOI: 10.1039/ d0ma00991a correct the shortcomings of using essential oils. The benefits of using polymer particles stem from the tailorable pore size and particle size, which can control the rate of release of the essential oil.

Thiol-ene based polymers show promise for drug delivery applications. They display distinct advantages such as delayed gel points with high monomer transformation, controlled mechanical and structural properties, and almost no oxygen inhibition. ${ }^{8}$ The advantages of the thiol-ene based polymers come from a step-growth free-radical mechanism. A thiyl radical is generated from the abstraction of a hydrogen by the photoinitiator. This radical then adds across the double bond of an alkene monomer generating a $\beta$-thioether carboncentered radical; the latter radical rapidly undergoes chain transfer with an additional thiol to regenerate the thiyl radical species. The thio-ether radical, in the absence of the propagation step, chain transfers to another thiol monomer. The coupling of two radicals terminates the reaction. Oxygen does not inhibit the reaction; instead, the oxygen molecule produces a peroxy radical that then chain transfers with a thiol, 
regenerating the propagating species. ${ }^{8-10}$ The thiol-ene reaction is probably best known for the formation of near-perfect crosslinked polymer networks, wherein polymerization with high conversion is reached before gelation and results in a homogeneous medium to produce equally crosslinked networks with well-defined physical and mechanical properties. ${ }^{8-10}$ By varying the numerous combinations of different multifunctional thiol and alkene monomers, the mechanical properties and distance between crosslinks for these systems can be easily tuned. Recent work has shown the viability to use thiol-ene systems via either suspension or emulsion polymerizations to create particles with controllable sizes. $^{11-16}$

Durham et al. were the first to show that a waterborne photopolymerization reaction could occur between a multifunctional alkene and thiol monomers to create particles. They used a suspension polymerization with sodium dodecyl sulfate (SDS) surfactant to react a 3-functional alkene to a 4-functional thiol with toluene or chloroform as the diluent. They were able to synthesize particles with a size range of 10 to $200 \mu \mathrm{m}$ by altering the rate of stirring, the ratio of solvent to monomers, and the amount of surfactant. ${ }^{11}$ Zhang et al. used a similar synthesis route, but incorporated a linear poly(methylmethacrylate) molecule to disrupt the crosslinking of the thiol-ene network. The work resulted in particles that were larger than $200 \mu \mathrm{m}$ in diameter and with controllable pore sizes. ${ }^{13} \mathrm{D}$. V. Amato et al. built on Durham et al.'s work to demonstrate the viability of using the mini-emulsion technique to create nanometer-sized particles with excess functional groups on the surface of the particles that could be used for subsequent functionalization. ${ }^{14}$ D. N. Amato et al. further expanded on this work to show that thiol-ene mini-emulsions could be used to encapsulate thymol dissolved in carvacrol. They further demonstrated that an alkene functionalized surfactant could be reacted into the particles to minimize the amount of surfactant needed to be removed. ${ }^{15}$ More recently, Shipp et al. demonstrated the viability to form linear and lightly crosslinked emulsions via thiol-ene reaction. ${ }^{16}$ While these examples are specific to the work presented in this paper, a more thorough review of particles formed via thiol- $x$ chemistries can be found elsewhere. ${ }^{17}$

While the previous studies with photo-initiated waterborne thiol-ene polymerizations have established synthesis routes to form controllable particle sizes and tailorable pore sizes, the bulk of these studies either focused on micro and mini-emulsions, have not investigated the ability of the formed particles to carry a drug payload, use harsh solvents, or cannot remove the stabilizing surfactant. Our work builds on previous research while addressing these deficiencies by using canola oil as the diluent solvent, altering the monomer and surfactant ratio while maintaining 1:1 functional group stoichiometry to control particle size, efficiently removing excess reactants and surfactant, and demonstrating tailorable release profiles for thymol.

\section{Materials and methods}

\section{General thiol-ene particle synthesis via asuspension}

The organic oil phase consisted of trimethylolpropane diallyl ether (2-ene) (Sigma-Aldrich, St. Louis, MO), pentaerythritol tetra(3-mercaptopropionate) (4-thiol) (Sigma-Aldrich, St. Louis, MO), dithiol polyethylene glycol (2-thiol) (Sigma-Aldrich, St. Louis, MO), and a photoinitiator (1-hydroxycyclohexyl phenyl ketone, commonly known by Irgacure 184) (Sigma-Aldrich, St. Louis, MO) and a diluent solvent (Fig. 1). Chemical composition was altered by changing the ratio of the 2-ene to the Hitenol KH (Motello Inc., Tulsa, OK) surfactant functional group ratios from $1.0: 0.0$ to $0.5: 0.5$ in 0.1 increments and by changing the ratio of 2 -thiol to 4 -thiol functional groups from $0.9: 0.1$ to $0.5: 0.5$ in 0.1 increments. A $1: 1$ stoichiometry of the $\mathrm{SH}$ and ene functional groups was maintained for all formulations by increasing the amount of 1-ene Hitenol $\mathrm{KH}$ as the amount 2-ene is lowered. The full list of the resulting 30 formulations used in this study can be seen in the ESI $\dagger$ (Fig. S1). These formulations allowed for the systematic alteration in both chemical structures, as well as the amount of crosslinking present in the particles. The water phase consisted of de-ionized (DI) water and Hitenol $\mathrm{KH}$ (25 wt\% in water). After adding the organic phase to surfactant/water, the resulting suspension was stirred at either $600 \mathrm{rpm}$ (hereafter referred as half-speed suspension, HSS) or $1200 \mathrm{rpm}$ (hereafter referred as full-speed suspension, FSS) for 10 minutes on a stir plate (Corning PC-220, Corning, NY). The emulsified solution was then cured using UV light $\left(365 \mathrm{~nm}, 40 \mathrm{~mW} \mathrm{~cm}^{-2}\right.$, Omnicure S1500, Ecelitas, Waltham, MA) for 10 minutes.

\section{Synthesis of thiol-ene particles with entrapped thymol}

The microsphere synthesis was performed as stated above; however, the organic oil phase included either butyl acetate (Sigma-Aldrich, St. Louis, MO) to create a control or thymol (Sigma-Aldrich, St. Louis, MO) and canola oil (Kroger, Cincinnati, $\mathrm{OH})$ (30 wt\%). Thymol-containing particles did not form without the canola oil diluent, instead they formed irregular shaped materials that stuck to the glass container. Thymol was dissolved in canola oil by heating to $95{ }^{\circ} \mathrm{C}$ for 1 minute. The formed microspheres were centrifuged at $2000 \mathrm{rpm}$ for 5 minutes, the liquid was decanted, and $10 \mathrm{~mL}$ of DI water was added. This process was repeated three times to isolate the particles from any excess reagents. Further purification was

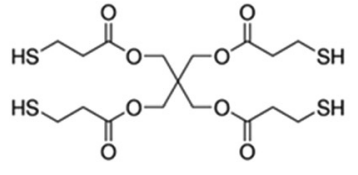

4-thiol

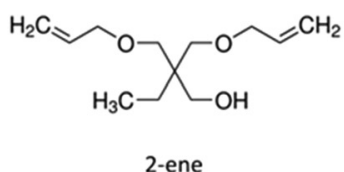

2-ene<smiles>SCCOCCOCCS</smiles>

2-thiol

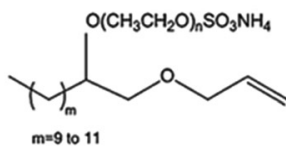

Hitenol-KH

Fig. 1 Chemical structures of the monomers used to create particles. 
achieved by soaking the particles for 24 hours in DI water, followed by one last centrifugation step. The residual reagent removal was confirmed to be $99 \%$ by Nanodrop UV-Vis spectrophotometer (ND-1000, Thermo Scientific, Waltham, MA) (Fig. S2, ESI $\dagger$ ). Formulations with a 2-thiol to 4-thiol ratio of $0.3: 0.7,0.4: 0.6$ and $0.5: 0.5$ were too delicate and did not survive the centrifugation steps.

\section{Optical microscopy and particle size analyses}

A Keyence VHX microscope (Keyence, Osaka, Japan) was used to image the particles. The samples were placed into a 24 well cell culture plate and imaged at various magnifications. ImageJ version $1.51 \mathrm{k}(\mathrm{NIH})$ was used for particle size analysis using the ellipse measurement tool. The minimum sample size was established as 100 particles to ensure an accurate representation of the population. The results of the particle analysis are reported as the mean diameter $\pm 95 \%$ confidence interval.

\section{Thymol release measurements}

After synthesis and purification, the particles were placed in a $25 \mathrm{~mL}$ scintillation vial with $10 \mathrm{~mL}$ of PBS. The Nanodrop UVVis spectrophotometer (ND-1000) was used to measure the release of thymol from the particles. $3 \mu \mathrm{L}$ of solution was placed on the ND-1000 pedestal, and the absorbance at $275 \mathrm{~nm}$ was measured. Drug release was measured for ten days, and the release plots were fit to the Peppas-Salhin equation. A 100\% loading efficiency was assumed for release calculations. Results are reported as the mean of three samples per formulation $\pm 95 \%$ confidence interval.

\section{Results and discussion}

\subsection{Particle synthesis and characterization}

Polymerizations without the surfactant resulted in the formation of large bulk polymers that tended to agglomerate onto the stir bar (Fig. 2a). While the result was not the formation of particles, the bulk polymer still encapsulated the thymol/canola oil, as indicated by the drug release (Fig. 3). However, the bulk polymers released minimal amount of thymol and their release profiles were not statistically significant from each other from days 1 to 4 . The percent of thymol released on day 4 were $1.5 \pm 0.7 \%$ for the 2 -ene ${ }_{1}$ Hitenol $_{0} 2$-thiol ${ }_{0.1} 4$-thiol ${ }_{0.9}$ formulation and $3.0 \pm 0.4 \%$ for the 2 -ene ${ }_{1}$ Hitenol $_{0} 2$-thiol ${ }_{0.2} 4$-thiol th. $_{0.8}$ formulation $(p>0.05)$. Overall, only $\sim 3 \%$ thymol was released by day 11 for each formulation (Fig. 2). Nevertheless, these findings demonstrate that the bulk reaction could be used to make drug-loaded materials and coatings that could be potentially molded into any desired shape.

We used two purification processes to remove the excess reagents. The first was to centrifuge the reacted particles using seven centrifugation cycles described in the methods section. The second was to centrifuge for three cycles and soak the particles for 24 hours in DI water before performing one final centrifugation cycle. Both processes resulted in 99\% removal of unreacted reagents (Fig. S3, ESI $\dagger$ ). Due to the delicate nature of
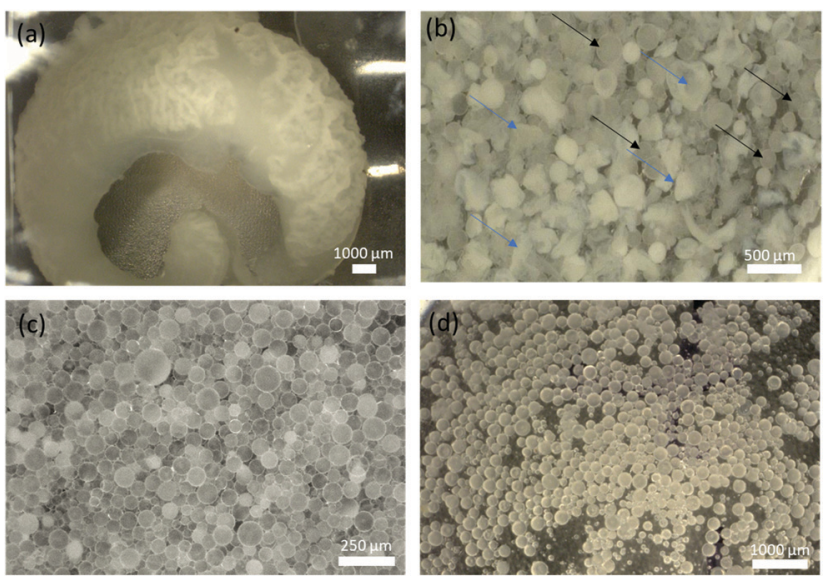

Fig. 2 Images of polymer particles. (a) Surfactant was needed to stabilize the monomers into droplets before polymerization, or the result was the formation of a bulk polymer. (b) Formulations with a 2 to 4 -thiol ratio below 0.7 resulted in particles that were too delicate to survive purification. Black arrows indicate intact particles that appear to be slightly transparent, blue arrows show damaged particles that appear to be like popped balloons and are more opaque. (c) Particles formed using butyl acetate as a control without any thymol. (d) Using canola oil as the diluent solvent resulted in the successful creation of thymol-containing particles.

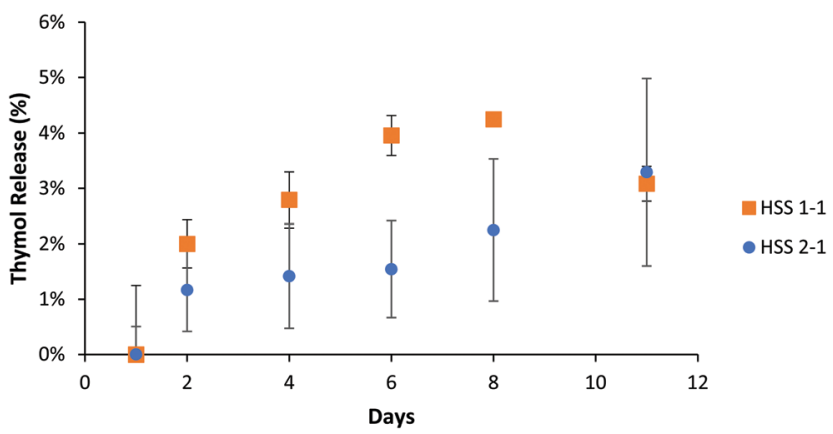

Fig. 3 Thymol release for the two formulations without the Hitenol $\mathrm{KH}$ using half speed suspension polymerization (HSS). Error bars represent 95\% confidence intervals (C.I.).

the particles, seven centrifugation cycles sometimes resulted in a portion of the particles becoming broken, so the fewer centrifugation cycles with soaking was preferred. Table 1 contains the formulations that survived the purification process. After purification, the control particles prepared using butyl acetate and no thymol were soaked in PBS for five days, and the absorbance was measured to determine if any reagents leached from the particles (Fig. S4a, ESI $\dagger$ ). A small amount of butyl acetate was observed in the PBS, as indicated by the UV-Vis spectrum matching that of butyl acetate (Fig. S4b and S5, ESI $\dagger$ ). During purification, it was found that the formulations with a 2-thiol to 4-thiol ratio of $0.3: 0.7,0.4: 0.6,0.5: 0.5$ (Fig. S1, ESI $\dagger$ ) resulted in particles that did not survive the purification process (Fig. 2b) as the particles were too delicate, likely due to the lower amount of the 4-thiol crosslinker.

Fig. 2b shows that the broken particles formed a core-shell architecture where the polymer entraps the bulk of the thymol/ 
Table 1 Polymer formulations that resulted in particles that were robust enough to survive the synthesis and purification process. It can be seen that functional group stoichiometry was maintained by increasing the amount of 1-ene Hitenol $\mathrm{KH}$ as the amount of 2-ene is decreased

\begin{tabular}{|c|c|c|c|c|c|c|}
\hline \# & $\begin{array}{l}\text { Ene: Hitenol func- } \\
\text { tional group ratio }\end{array}$ & $\begin{array}{l}\text { 2-Ene } \\
(\mu \mathrm{L})\end{array}$ & $\begin{array}{l}\text { 2-Thiol } \\
(\mu \mathrm{L})\end{array}$ & $\begin{array}{l}\text { 4-Thiol } \\
(\mu \mathrm{L})\end{array}$ & $\begin{array}{l}\text { Hitenol } \\
(\mathrm{mL})\end{array}$ & $\begin{array}{l}\text { Water } \\
(\mathrm{mL})\end{array}$ \\
\hline \multicolumn{7}{|c|}{ 2-Thiol : 4-thiol functional group ratio $0.1: 0.9$} \\
\hline 1 & $1.0: 0.0$ & 89.0 & 6.4 & 272.5 & 0.0 & 10 \\
\hline 2 & $0.9: 0.1$ & 78.5 & 6.4 & 272.5 & 0.4 & 9.6 \\
\hline 3 & $0.8: 0.2$ & 68.1 & 6.4 & 272.5 & 0.8 & 9.2 \\
\hline 4 & $0.7: 0.3$ & 57.6 & 6.4 & 272.5 & 1.2 & 8.8 \\
\hline 5 & $0.6: 0.4$ & 47.1 & 6.4 & 272.5 & 1.6 & 8.4 \\
\hline 6 & $0.5: 0.5$ & 36.6 & 6.4 & 272.5 & 2.0 & 8 \\
\hline \multicolumn{7}{|c|}{ 2-Thiol : 4-thiol functional group ratio $0.2: 0.8$} \\
\hline 1 & $1.0: 0.0$ & 89.0 & 12.8 & 242.3 & 0.0 & 10 \\
\hline 2 & $0.9: 0.1$ & 78.5 & 12.8 & 242.3 & 0.4 & 9.6 \\
\hline 3 & $0.8: 0.2$ & 68.1 & 12.8 & 242.3 & 0.8 & 9.2 \\
\hline 4 & $0.7: 0.3$ & 57.6 & 12.8 & 242.3 & 1.2 & 8.8 \\
\hline 5 & $0.6: 0.4$ & 47.1 & 12.8 & 242.3 & 1.6 & 8.4 \\
\hline 6 & $0.5: 0.5$ & 36.6 & 12.8 & 242.3 & 2.0 & 8 \\
\hline
\end{tabular}

canola oil. Butyl acetate was used to prepare control particles (Fig. 2c) as it has been successfully used previously in creating polymer particles and has a limited solubility in water at $6.8 \mathrm{mg} \mathrm{mL}^{-1}$. The control particles were used to show that the monomer combinations would result in the removal of unreacted reagents, and to show that the particles are not leaching reagents over time. The same formulations were made using thymol dissolved in canola oil as a diluent, which resulted in the successful creation of thymol-containing polymer particles (Fig. 2d). There have been several previous studies that have reacted thiols to the alkene in presence of fatty acids using UV light free radical reactions to create coatings. These studies have shown that the fatty acids have very low reactivity towards thiols and need very specific conditions to react; namely, a thiol to ene ratio greater than 3, negative temperatures to decrease the faster thiolthiol coupling reaction, large amounts of photoinitiator $(0.1: 1$ initiator molecule to alkene group), a nitrogen atmosphere to prevent competitive oxidation, hours to days to reach low to high conversions, and/or chemical modification of the fatty acid prior to the thiol-ene reaction. Given that our reactions happen in 10 minutes at ambient conditions, we suspect there to be negligible reaction between the thiol monomers and the alkene group of the canola oil. ${ }^{18-20}$

Fig. 4 shows the resulting particle sizes for the formulations varied with the thiol, ene, and surfactant composition. Overall, the higher amount of 4-thiol results in statistically larger particles. As shown in Fig. 4, all 0.1:0.9 2-thiol to 4-thiol formulations yield larger particles than the $0.2: 0.8$ formulations $(p \leq 0.05)$. For example, with the control particles, the 2-thiol to 4-thiol ratio of 0.1:0.9 uses the higher amount of 4-thiol and results in larger mean particle diameter $(161 \pm 3 \mu \mathrm{m}$,

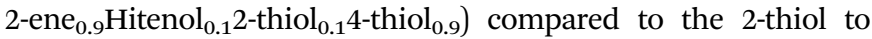
4 -thiol ratio of $0.2: 0.8$, which uses lower amount of 4-thiol and results in a smaller mean particle diameter $(92 \pm 2 \mu \mathrm{m}$,

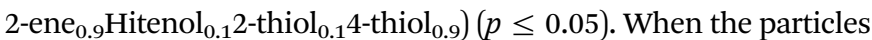
are synthesized with thymol and canola oil instead of butyl acetate at $1200 \mathrm{rpm}$ (FSS) the same trends hold. When 2-ene ${ }_{0.5} \mathrm{Hitenol}_{0.5}$ 2-thiol ${ }_{0.1} 4$-thiol $_{0.9}$, the formulation results in a mean particle
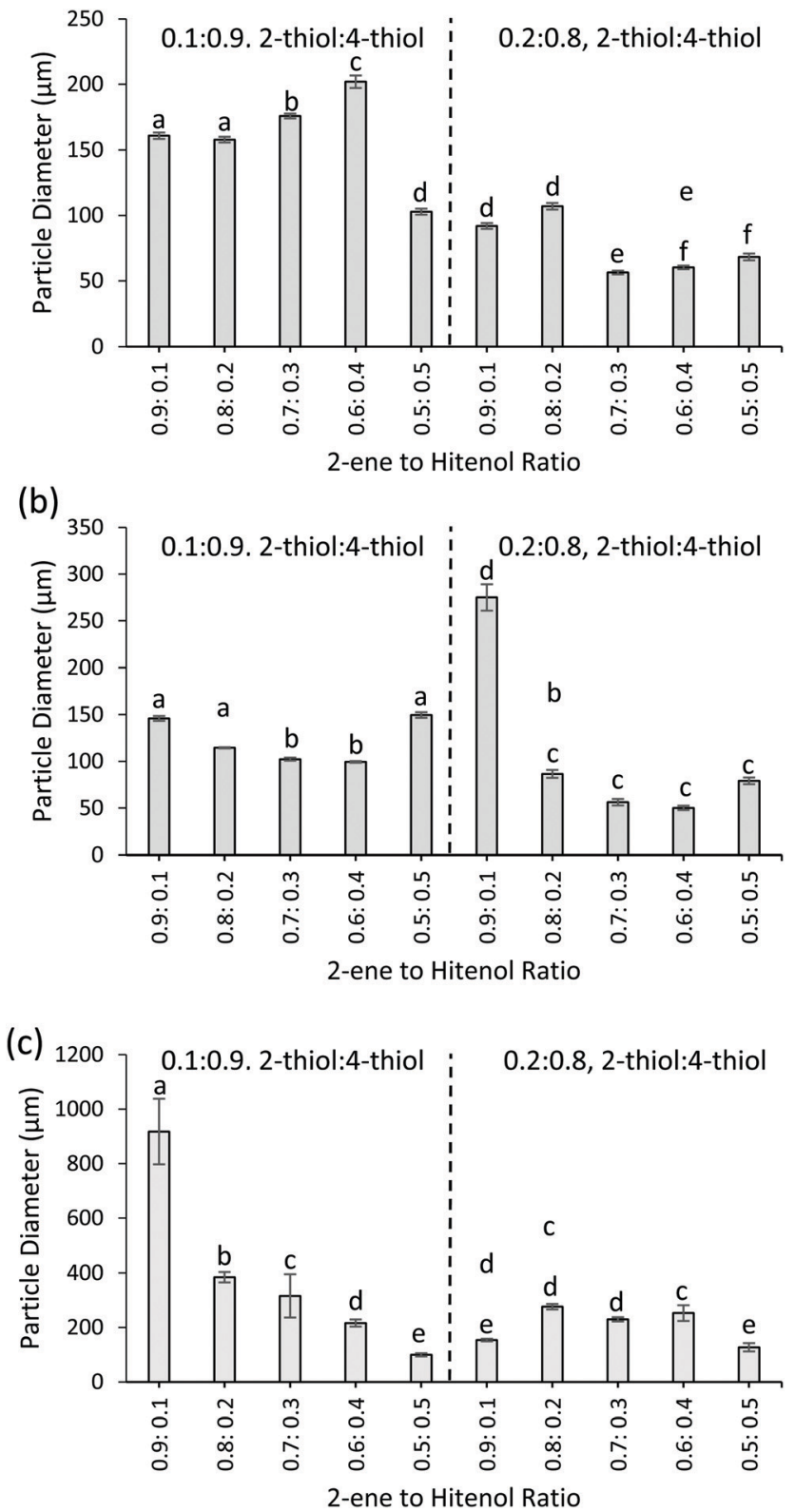

Fig. 4 Particle sizes (a) butyl acetate, (b) thymol/canola oil stirred at $1200 \mathrm{rpm}$, and (c) thymol/canola oil stirred at $600 \mathrm{rpm}$. Letters indicate statistical groupings. Different letters show that the values are significantly different $(p \leq 0.05)$. Error bars represent 95\% C.I.

diameter of $149 \pm 3 \mu \mathrm{m}$ and when the amount of 4-thiol is decreased 2 -ene ${ }_{0.5} \mathrm{Hitenol}_{0.5} 2$-thiol ${ }_{0.2} 4$-thiol ${ }_{0.8}$ the particle diameter decreases to $79 \pm 4 \mu \mathrm{m}$. When the stirring speed is decreased for the thymol and canola oil particles to $600 \mathrm{rpm}$ (HSS), the mean diameters increase. This can be seen by comparing the mean diameter for the 2-thiol to 4 -thiol samples with a ratio of $0.1: 0.9$ and $0.2: 0.8$ for both HSS and FSS. An example is 2-ene ${ }_{0.8} \mathrm{Hitenol}_{0.2} 2$-thiol $_{0.1} 4$-thiol - $_{0.9}$, where the HSS reaction resulted in a mean particle diameter of $384 \pm 19 \mu \mathrm{m}$ compared to the $114 \pm 1 \mu \mathrm{m}$ particles obtained in the FSS reaction. The slower stirring was expected to result in larger particles, as this has been demonstrated before by Durham et al. ${ }^{11}$ 


\subsection{Thymol release}

Thymol has a maximum UV absorbance at $275 \mathrm{~nm}$, which overlaps the Hitenol KH surfactant, 2-thiol, 4-thiol, and 2-ene monomer (Fig. S5, ESI $\dagger$ ). Recalling that the particles were successfully purified (Fig. S2 and S3, ESI $\dagger$ ) and that the maximum absorbance at $275 \mathrm{~nm}$ for the control particles did not significantly change over five days (Fig. S4, ESI $\dagger$ ), any increase in the absorbance measured at $275 \mathrm{~nm}$ for the thymol-loaded particles must indicate released thymol and not excess monomer or surfactant. A previous study that used a thymol/carvacrol loaded particles formed by mini-emulsion polymerization examined the release of the essential oils using gas chromatography/mass-spectroscopy. The particles were placed in DI water, and the release was monitored for 24 hours. The amount released immediately hit the solubility limit of thymol in the water at $0.85 \mathrm{mg} \mathrm{mL}^{-1}$ at 0 hours, and no further release was observed past $3 \%$ of the total loaded thymol/carvacrol. ${ }^{15}$ Our research accounts for the solubility limit of thymol in water by placing the particles in excess of PBS solution so that the solubility limit is never reached. Thymol is known to have a linear solubility in water; therefore, the standard curve for this study involved measuring thymol at $0.85 \mathrm{mg} \mathrm{mL}^{-1}$ (Fig. S5, ESI $\dagger$ ) and taking that as being $100 \%$ drug release. We observed a gradual thymol release up to $62 \%$ by ten days depending on the formulation for the reactions stirred at $600 \mathrm{rpm}$ (Fig. 5a).
The formulations stirred at $1200 \mathrm{rpm}$ exhibited faster release with the 2-ene ${ }_{0.5}$ Hitenol $_{0.5} 2$-thiol ${ }_{0.2} 4$-thiol ${ }_{0.8}$ formulation showing a burst release with $95 \%$ of thymol released in one day (Fig. 5b). In fact, the 2-ene ${ }_{0.5} \mathrm{Hitenol}_{0.5} 2$-thiol ${ }_{0.2} 4$-thiol ${ }_{0.8}$ formulation showed the fastest drug release for both synthesis methods at $95 \%$ release for the FSS method at day 1 and $54 \%$ release for the HSS method by day 10 (Fig. 4). The 2-ene ${ }_{0.5}$ Hitenol $_{0.5} 2$-thiol ${ }_{0.2} 4$-thiol ${ }_{0.8}$ formulation has the lowest amount of 4-thiol and thus the lowest amount of crosslinking; it also has the highest amount of surfactant (Table 1). Note that the Hitenol KH surfactant is a mono-functional alkene (Fig. 1) that can react with the particles and limit the amount of crosslinking.

\subsection{Modeling of drug release}

The drug release plots were fit to several models, but ultimately the Peppas-Sahlin equation was chosen because it had the highest $R^{2}$. The Peppas-Sahlin equation where $M_{\mathrm{t}} / M_{\mathrm{inf}}=$ $k_{1} t^{m}+k_{2} t^{2 m}$ is useful because it can be used on drug delivery vehicles with varying geometries, and it can be used to model the amount of Fickian diffusion versus super case II for drug release. The super case II release mechanism is a result of physical processes that occur in the polymer membrane, such as chain relaxation and mobility, and erosion of the membrane. $^{22}$ This is performed using the $k_{1}$ and $k_{2}$ terms

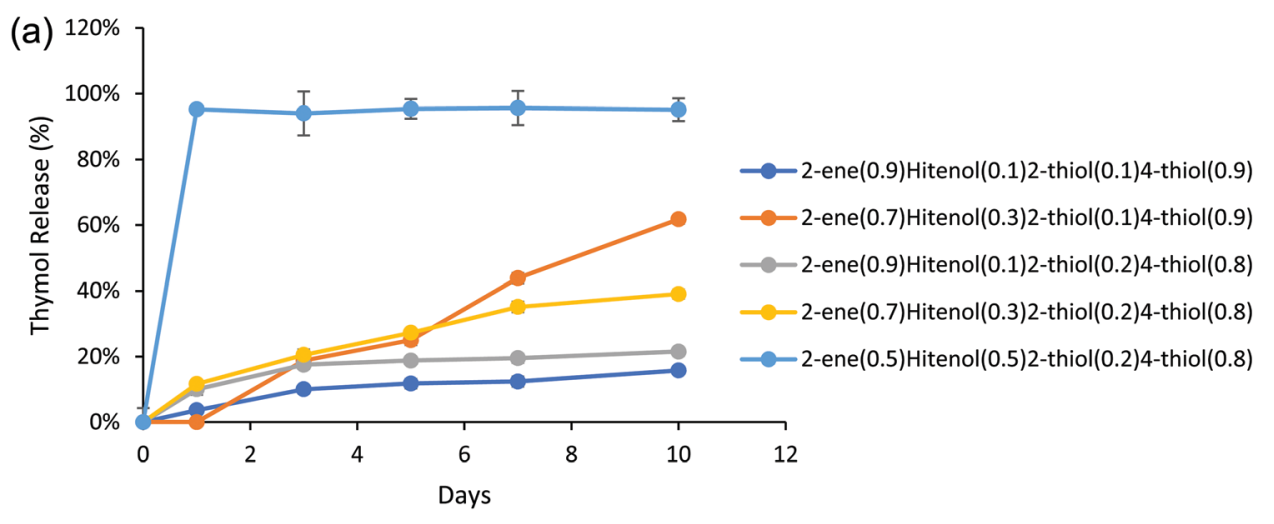

(b)

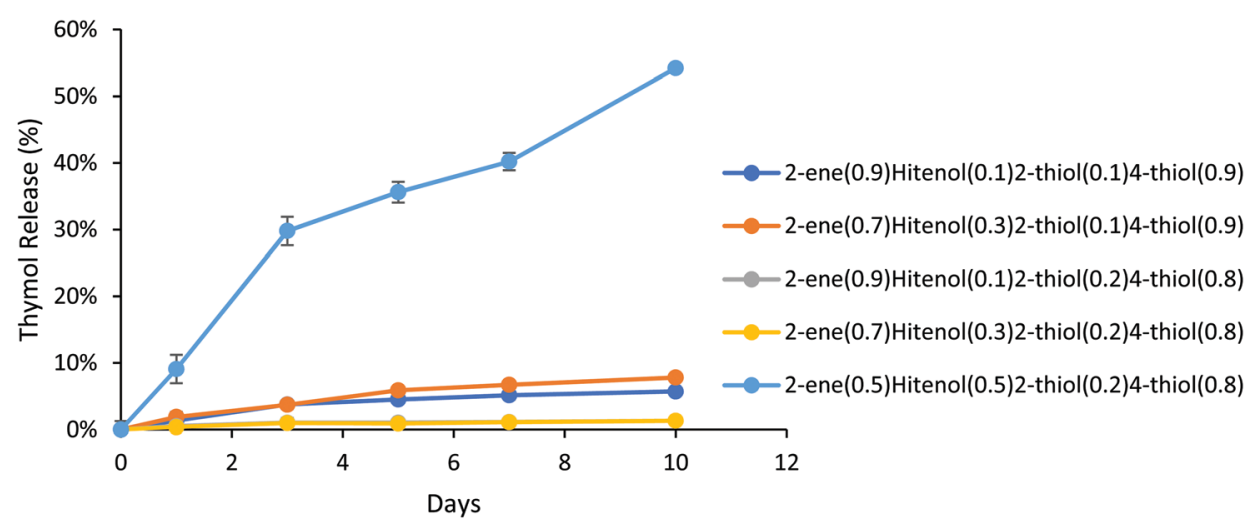

Fig. 5 Drug release profiles for thymol/canola oil loaded particles. The particles stirred at $600 \mathrm{rpm}$ (a) have slower release profiles than the particles synthesized with a stirring speed of 1200 rpm (b). Error bars represent 95\% C.I. 
Table 2 The values for the Peppas-Sahlin equation. The values for the Peppas-Sahlin equation, and the calculated values for $F$, the percent Fickian diffusion

\begin{tabular}{|c|c|c|c|c|c|c|c|c|c|c|}
\hline$\frac{\text { Formulation }}{\text { Synthesis }}$ & HSS & FSS & HSS & FSS & HSS & FSS & HSS & FSS & HSS & FSS \\
\hline$F$ at 1 day & 0 & 0 & 0 & 0 & 0 & 0 & 0 & 0 & 0 & 0.01 \\
\hline Slope & 0.0249 & 0.0007 & 0.0331 & 0.2512 & 0.0052 & 0.0872 & 0.0052 & 0.1626 & 0.2211 & 0.3261 \\
\hline Intercept & -0.0006 & 0 & -0.0028 & -0.1 & 0 & 0.024 & 0 & -0.0036 & -0.0261 & 0.3386 \\
\hline Hixon-Crowell $R^{2}$ & 0.61 & 0.61 & 0.65 & 0.84 & 0.52 & 0.48 & 0.57 & 0.62 & 0.67 & 0.31 \\
\hline
\end{tabular}

that are calculated by fitting the drug release data and the equation $F=\frac{1}{1+\frac{k 2}{k 1} t^{m}}$, which gives the percent of Fickian diffusion. ${ }^{21}$ The Fickian diffusion mechanism is driven by thermodynamic forces such as concentration gradients and chemical potential and typically exhibits near-constant diffusion of the eluents that follow Fick's first and second laws of diffusion. ${ }^{22}$ The value for $m$ was chosen as 0.43 for a sphere. ${ }^{21}$ The calculated values for $F$ were zero or very close to zero, indicating that the mechanism for drug release was not Fickian diffusion, but super case II (Table 2).

The Hixon-Crowell cubic root equation and UV-Vis spectroscopy was used to determine if erosion of the particle was part of the release mechanism. This equation is specifically designed to evaluate the drug release for systems where the surface area and diameter is changing from erosion or swelling/ shrinking. ${ }^{23}$ The low-moderate $R^{2}$ values for the Hixon-Crowell equation indicate that erosion is not a primary mechanism for thymol release for these systems (Table 2). This is further confirmed by Nanodrop UV-Vis spectroscopy that indicates no change in the spectra for the control particles over five days (Fig. S5, ESI $\dagger$ ). While erosion may not be a primary mechanism, the low-moderate $R^{2}$ for Hixon-Crowell may indicate that a change in diameter likely plays a part in the diffusion mechanism of thymol release.

\section{Conclusions}

Suspension polymerization using thiol-ene click chemistry was used successfully to create a variety of particle sizes that encapsulated thymol. Canola oil was used as a biologically compatible alternative to the harsh organic solvents used in previous studies. Canola oil could be used in future studies to dissolve any lipophilic drug. A variety of particle sizes were made that ultimately depended on the stirring speed with the faster speed resulting in smaller particles. Thymol release could be controlled by altering the monomer ratios used in the polymerization with more crosslinker and less surfactant leading to slower release and less crosslinker and more surfactant leading to faster thymol release. The Peppas-Sahlin equation was used to model the thymol release and indicated that the release mechanism was super case II, which depends more on polymer chain movements than traditional Fickian diffusion. Taken together, this research demonstrates the viability of using thiol-ene suspension polymerization and canola oil as a diluent to create particles that can carry lipophilic drugs.

\section{Funding}

This work was partially funded by the National Institutes of Food and Agriculture: United States Department of Agriculture Exploratory Research Grant (Award \# 2018-67031-27502) awarded to AVJ, and the National Institutes of Food and Agriculture: United States Department of Agriculture Predoctoral Fellowship (Award \# 2019-67011-29520) awarded to JSC.

\section{Conflicts of interest}

The authors have no conflicts of interest to disclose.

\section{Acknowledgements}

The authors thank Montello Inc. for the kind donation of Hitenol KH. Jui Gaikwad and Joshua Michael participated in the Undergraduate and Professional Student Training in Advanced Research Techniques (UPSTART) Program. Chipo Chapusha participated in the Summer Undergraduate Research Experience (SURE) Program.

\section{References}

1 A. Yorgancioglu and E. E. Bayramoglu, Production of cosmetic purpose collagen containing antimicrobial emulsion with certain essential oils, Ind. Crops Prod., 2013, 44, 378-382.

2 M. B. Isman, S. Miresmailli and C. Machial, Commercial opportunities for pesticides based on plant essential oils in agriculture, industry and consumer products, Phytochem. Rev., 2011, 10, 197-204.

3 J. Tian, X. Ban, H. Zeng, B. Huang, J. He and Y. Wang, In vitro and in vivo activity of essential oil from dill (Anethum graveolens L.) against fungal spoilage of cherry tomatoes, Food Control, 2011, 22, 1992-1999. 
4 J. Gutierrez, C. Barry-Ryan and P. Bourke, The antimicrobial efficacy of plant essential oil combinations and interactions with food ingredients, Int. J. Food Microbiol., 2008, 124, 91-97.

5 C. D. Bishop, Antiviral activity of the essential oil of Melaleuca alternifolia (maiden amp; Betche) Cheel (tea tree) against tobacco mosaic virus, J. Essent. Oil Res., 1995, 7, 641-644.

6 A. Marchese, I. E. Orhan, M. Daglia, R. Barbieri, A. Di Lorenzo, S. F. Nabavi, O. Gortzi, M. Izadi and S. M. Nabavi, Antibacterial and antifungal activities of thymol: A brief review of the literature, Food Chem., 2016, 210, 402-414.

7 L. Sánchez-González, M. Vargas, C. González-Martínez, A. Chiralt and M. Chafer, Use of essential oils in bioactive edible coating:s: a review, Food Eng. Rev., 2011, 3, 1-6.

8 C. E. Hoyle and C. N. Bowman, Thiol-ene click chemistry, Angew. Chem., Int. Ed., 2010, 49, 1540-1573.

9 A. B. Lowe, Thiol-ene "click" reactions and recent applications in polymer and materials synthesis: a first update, Polym. Chem., 2014, 5, 4820-4870.

10 M. J. Kade, D. J. Burke and C. J. Hawker, The power of thiolene chemistry, J. Polym. Sci., Part A: Polym. Chem., 2010, 48, 743-750.

11 O. Z. Durham, S. Krishnan and D. A. Shipp, Polymer Microspheres Prepared by Water-Borne Thiol-Ene Suspension Photopolymerization, ACS Macro Lett., 2012, 1, 1134-1137.

12 C. Wang, M. Podgórski and C. N. Bowman, Monodisperse functional microspheres from step-growth "click" polymerizations: preparation, functionalization and implementation, Mater. Horiz., 2014, 1, 535-539.

13 J. Tan, C. Li, J. Zhou, C. Yin, B. Zhang, J. Gu and Q. Zhang, Fast and facile fabrication of porous polymer particles via thiol-ene suspension photopolymerization, RSC Adv., 2014, 4, 13334-13339.
14 D. V. Amato, D. N. Amato, A. S. Flynt and D. L. Patton, Functional, sub-100 nm polymer nanoparticles via thiol-ene miniemulsion photopolymerization, Polym. Chem., 2015, 6, 5625-5632.

15 D. N. Amato, D. V. Amato, O. V. Mavrodi, D. A. Braasch, S. E. Walley, J. R. Douglas, D. V. Mavrodi and D. L. Patton, Destruction of opportunistic pathogens via polymer nanoparticle-mediated release of plant-based antimicrobial payloads, Adv. Healthcare Mater., 2016, 5, 1094-1103.

16 O. Z. Durham, D. V. Chapman, S. Krishnan and D. A. Shipp, Radical mediated thiol-ene emulsion polymerizations, Macromolecules, 2017, 50, 775-783.

17 Olivia Z. Durham and Devon A. Shipp, Polymer Colloids from Step-Growth Thiol-X Polymerizations, Polym. Rev., 2020, 1-26.

18 Prakash Alagi, et al., Efficient and quantitative chemical transformation of vegetable oils to polyols through a thiolene reaction for thermoplastic polyurethanes, Ind. Crops Prod., 2016, 87, 78-88.

19 Yu Hui Zhao, et al., Thiol-ene chemistry of vegetable oils and their derivatives under UV and air: a model study by using infrared spectroscopy and mass spectrometry, RSC Adv., 2017, 76, 3343-3352.

20 M. Desroches, et al., Synthesis of bio-based building blocks from vegetable oils: A platform chemicals approach, Lipid Technol., 2014, 26.2, 35-38.

21 N. A. Peppas and J. J. Sahlin, A simple equation for the description of solute release. III. Coupling of diffusion and relaxation, Int. J. Pharm., 1989, 57, 169-172.

22 P. I. Lee, Kinetics of drug release from hydrogel matrices, J. Controlled Release, 1985, 2, 277-288.

23 M. A. Kalam, M. Humayun, N. Parvez, S. Yadav, A. Garg, S. Amin, Y. Sultana and A. Ali, Release kinetics of modified pharmaceutical dosage forms: a review, Cont. J. Pharm. Sci., 2007, 1, 30-35. 